\title{
Set-Theoretic Dependence
}

\author{
John Wigglesworth \\ Centre for Philosophy of Natural and Social Science \\ London School of Economics
}

\begin{abstract}
In this paper, we explore the idea that sets depend on, or are grounded in, their members. It is said that a set depends on each of its members, and not vice versa. Members do not depend on the sets that they belong to. We show that the intuitive modal truth conditions for dependence, given in terms of possible worlds, do not accurately capture asymmetric dependence relations between sets and their members. We extend the modal truth conditions to include impossible worlds and give a more satisfactory account of the dependence of a set on its members. Focusing on the case of singletons, we articulate a logical framework in which to evaluate set-theoretic dependence claims, using a normal first-order modal logic. We show that on this framework the dependence of a singleton on its single members follows from logic alone. However, the converse does not hold.
\end{abstract}

It is natural to think of dependence in terms of necessity. One thing depends on another because it is necessary that, given the one exists, the other exists as well. In other words, you can't have the one without the other. It is also natural, though perhaps less so, to think that a set depends on, or is grounded in, its members, and not vice versa. The dependence between a set and its members is asymmetric. But these thoughts are incompatible.

They are incompatible because an account of dependence, articulated in terms of necessity, will yield a symmetric dependence relation between a

Australasian Journal of Logic (12:3) 2015, Article no. 2 
set and its members, assuming that necessity is given the usual possible worlds truth conditions. In this paper, we argue for a different account of the dependence that holds between a set and its members. This account is given in terms of worlds, but we appeal to both possible and impossible worlds. The nature of these impossible worlds will be discussed in what follows. But we will show that invoking impossible worlds yields an asymmetric dependence relation, such that sets depend on their members, but members do not depend on the sets they belong to.

In section 1, we look at why one might think that sets depend on their members. Section 2 presents the standard modal truth conditions for dependence, showing why they fail to generate asymmetric dependence relations between sets and their members. Section 3 presents alternative modal truth conditions for dependence that appeals to impossible worlds. In this section we also sketch the argument that these truth conditions accurately capture the asymmetric dependence of a set on its members, focusing on the case of singletons, i.e., sets with single members. Section 4 fills in the technical details of the argument that singletons depend on their members and that this dependence is asymmetric. Section 5 concludes with a discussion of the nature of this asymmetry. We also consider the possibility of extending this argument, beyond the case of singletons, to show that all sets depend on their members, and that this dependence is asymmetric.

\section{The Iterative Conception of Set}

In the literature on grounding and dependence, many assume, or at least grant the intuition, that sets depend on, or are grounded in, their members. They usually do so without any argument. ${ }^{1}$ Indeed, if sets depend on their members, they may do so in several ways. A set may depend on its members for its existence, or for its identity, or for some of its other

\footnotetext{
${ }^{1}$ See Cameron (2008), Correia (2005) Fine (1995), Lowe (2005), Paseau (2010), Potter (2004), Schaffer (2010), Schnieder (2007), Trogdon (2013), among others. I take "grounding" and "dependence" to be terms that describe the same relation: for some sense of "depends", $x$ depends on $y$ iff $x$ is grounded in $y$. Nothing turns on this choice. One may want to distinguish these terms, but discussion of the reasons for doing so need not be taken up here. The rest of the paper will be presented using the language of dependence.
}

Australasian Journal of Logic (12:3) 2015, Article no. 2 
properties, like its cardinality. It is the first of these that we are primarily interested in. The claim we explore is that the existence of a set depends on the existence of its members. In what follows, when we say that a set depends on its members, it is the existential sense of dependence that we are referring to.

So why think that the existence of a set depends on the existence of its members? There are two lines of thought here. The first is motivated by a general, non-technical conception of what a set is. According to this view, a set just is a collection of its members. If that's what a set is, then if the set exists, its members must exist as well. One can't collect together things that do not exist. In this sense, the existence of the set depends on the existence of its members. It must be that, if a set exists, then its members exist as well.

The second reason for thinking that sets depend on their members is motivated by a widely accepted view in the philosophy of mathematics. On this view, the correct mathematical conception of set is the iterative conception, according to which sets are built in stages. ${ }^{2}$ The iterative conception of set is a fairly natural conception. It is a conception that one could come up with in the absence of any extensive formal training in set theory. If we start from the idea that a set is simply a collection of objects, then the iterative conception suggests itself quite readily.

If sets are simply collections of objects, then for any things that exist, we should be able to take various sets that collect those things up in different ways. Even if we started with nothing at all, we should at least be able to take the set that contains nothing at all. And so we now have one set, the empty set $\varnothing$. We then take all of the various collections of everything that we have so far, giving us the empty set $\varnothing$ and its singleton $\{\varnothing\}$. And we keep doing this. Forever.

What results is called the cumulative hierarchy of sets. The cumulative hierarchy is divided into levels that correspond to each stage in the process we have described. Each level $V_{\alpha}$ of the hierarchy is defined using ordinal recursion:

\footnotetext{
${ }^{2}$ See Boolos (1971).
}

Australasian Journal of Logic (12:3) 2015, Article no. 2 


$$
\begin{aligned}
V_{0} & =\varnothing \\
V_{\alpha+1} & =\mathcal{P}\left(V_{\alpha}\right) \\
V_{\lambda} & =\bigcup_{\alpha<\lambda} V_{\alpha} \text { if } \lambda \text { is a limit ordinal }
\end{aligned}
$$

The cumulative hierarchy, which exhausts the universe of sets according to the iterative conception, can adequately serve as an ontological foundation for the rest of mathematics. That is, the rest of mathematics can be done from within set theory, and the cumulative hierarchy provides all of the mathematical objects that one needs to do so. But if the iterative conception is the correct mathematical conception of set, then these are all the sets there are. There are no sets other than those that show up at some level in the cumulative hierarchy.

Why think that this is the case? The question is legitimate because there are mathematically interesting sets that do not appear at any level in the cumulative hierarchy. Most notably, non-well-founded sets, including selfmembered sets, and sets that involve infinitely descending membership chains, are not in the hierarchy. On the iterative conception, there are no such sets. But there are theories of non-well-founded sets that are both elegant and useful. ${ }^{3}$

To justify the iterative conception as the correct mathematical conception of set, one occasionally sees reference to a relation of dependence that holds between a set and its members. Luca Incurvati articulates this position when he says, "Sometimes, the iterative conception is described simply as the conception of set one ends up with if one takes sets as bearing such a relation of priority or dependence between them" $(2012, p .5)$. This view is also described by Michael Potter, who explores the idea that "a fundamental relation of presupposition, priority, or ... dependence between collections" is implicit in the iterative conception (2004, p. 36, emphasis in original). The cumulative hierarchy just looks like a picture that invokes some notion of dependence. The cumulative hierarchy, at each level, uses sets that are available to construct further sets at the next level. Those sets could not be constructed if the objects at previous levels had not existed. Each set has as its members, and therefore depends on, sets that were created at previous levels.

These considerations about the nature of sets and the cumulative hierar-

\footnotetext{
${ }^{3}$ See Aczel (1988) and Barwise \& Moss (1996).
} 
chy do not constitute a well formed argument in favour of the claim that sets depend on their members. The first line of thought relies on vague intuitions about existence and the process of collecting objects together. The second line of thought, perhaps based in these vague intuitions, simply takes the claim that sets depend on their members, and uses it to motivate a conception of set that rules out certain mathematical objects, like nonwell-founded sets. In what follows, we evaluate several ways to make the claim that sets depend on their members more philosophically precise. This involves articulating and evaluating necessary and sufficient truth conditions for dependence. Once we have settled on appropriate truth conditions, the main argument that sets depends on their members is then located in the fact that it follows from these truth conditions.

\section{Modal Truth Conditions for Dependence}

Dependence, in its various forms, is a relation that plausibly holds between many entities, not just sets. It is the relation that plausibly holds between a whole and its parts, or between a hole and its host. It is the relation that, more contentiously, holds between moral facts and non-moral facts, or between modal facts and non-modal facts. Note that the truth conditions given here are only intended to apply to set-theoretic dependence, the dependence that holds between a set and its members. We make no claim that they generalise to capture dependence between other objects or other kinds of entities.

Dependence is generally thought to be an asymmetric relation: if $x$ depends on $y$, then $y$ does not depend on $x$. While some argument is surely needed for the claim that dependence is asymmetric in every case, it does seem that sets, in particular singletons, give one of the clearer cases of asymmetric dependence.

It is most common to try to understand dependence in modal terms. One thing depends on another when you can't have the one without the other. If we make this naive conception philosophically precise, we get intuitive modal truth conditions for dependence.

Modal Dependence. $x$ depends on $y$ iff in every metaphysically possible world

Australasian Journal of Logic (12:3) 2015, Article no. 2 
$w$, if $y$ doesn't exist at $w$, then $x$ doesn't exist at $w$.

Nearly all of the recent literature on dependence rejects these modal truth conditions for dependence. These conditions are rejected because they cannot accurately capture the asymmetric dependence of a singleton on its single member. They fail in this regard because a set exists in every metaphysically possible world that has all of its members, and so a singleton exists in every metaphysically possible world in which its single member exists. For any object $x, x$ and $\{x\}$ exists in all of the same metaphysically possible worlds. This symmetry is even more apparent when we consider pure sets. As purely mathematical objects, pure sets exist in every metaphysically possible world. So, for example, the empty set $\varnothing$ and its singleton $\{\varnothing\}$ exist in all of the same possible worlds, because they both exist in every possible world.

As a result we find that the sentence

In every metaphysically possible world $w, x$ exists at $w$ if and only if $\{x\}$ exists at $w$.

is true. It follows from the modal truth conditions that $x$ and $\{x\}$ symmetrically depend on each other. But this is not how we think dependence works. Dependence is generally thought to be a relation that is asymmetric. And even if there are plausible cases of symmetric dependence, it's not clear that the set-theoretic case is one of them. The dependence between a singleton and its member is generally taken to be such that the singleton depends on the member, and not vice versa.

There are, however, plausible set-theoretic examples where the modal truth conditions give the proper, expected, asymmetric dependence relations. Consider the pair set $\{x, y\}$, where $x$ and $y$ are two distinct contingent entities. As sets are taken to depend on each of their members, this set depends on $x$, and it depends on $y$. The strict conditionals

In every metaphysically possible world $w$, if $x$ doesn't exist at $w$, then $\{x, y\}$ doesn't exist at $w$.

In every metaphysically possible world $w$, if $y$ doesn't exist at $w$, then $\{x, y\}$ doesn't exist at $w$.

both seem true. If $x$ does not exist, then no set can have $x$ as a member. Similarly for $y$. And so in either case, nothing can be the set $\{x, y\}$. So that

Australasian Journal of Logic (12:3) 2015, Article no. 2 
set doesn't exist.

But the strict conditionals

In every metaphysically possible world $w$, if $\{x, y\}$ doesn't exist at $w$, then $x$ doesn't exist at $w$.

In every metaphysically possible world $w$, if $\{x, y\}$ doesn't exist at $w$, then $y$ doesn't exist at $w$.

both seem false. Concerning the first conditional, there is a world where the pair set fails to exist because $y$ doesn't exist, even though $x$ does. And analogously for the second conditional. So it is not that modal truth conditions always fail in the context of sets. Problems arise only when the relata of the dependence relation exist in all of the same possible worlds. This happens in the case of singletons and their members, and when the relata are necessarily existing objects. Dependence relations require one to discriminate between the existence of one relatum and the existence of the other. In these cases there simply aren't enough possible worlds to do this.

What we have here is a problem of hyperintensionality. Hyperintensional contexts occur when possible worlds are not enough to discriminate between two intuitively different things. In this regard, the dependence relation is hyperintensional. We want to be able to say that, for all $x$, the singleton of $x$ depends on $x$, and not vice versa. But $x$ and its singleton exist in all of the same possible worlds. There aren't enough possible worlds to make this distinction. This is even more obvious in the case of objects that exist in every possible world.

\section{Revised Truth Conditions for Dependence}

In the presence of hyperintensionality, a natural move is to extend the possible worlds framework with more worlds, so-called impossible worlds. This is the strategy we will pursue here, starting by modifying the truth conditions for set-theoretic dependence.

Revised Modal Dependence. $x$ depends on $y$ iff in every world $w$, either metaphysically possible or metaphysically impossible, if $y$ doesn't exist at $w$, then $x$ doesn't exist at $w$.

Australasian Journal of Logic (12:3) 2015, Article no. 2 
These truth conditions differ from the original modal account by allowing consideration of impossible worlds. But we have to be careful about which impossible worlds we allow under consideration. For our purposes, we allow metaphysically impossible worlds, but not logically impossible worlds. So the worlds under consideration will obey the laws of classical logic. And so we can reason about them and from within them in a coherent and familiar way. This choice is justified by considering that, in order to evaluate dependence claims, we shouldn't have to change any of the laws of logic, or consider situations (i.e., worlds) where the laws of logic fail.

We will, however, need to consider metaphysically impossible worlds. For example, if we were to evaluate the claim that $\{\varnothing\}$ depends on $\varnothing$, we consider what happens at worlds where $\varnothing$ fails to exist. These worlds will be metaphysically impossible worlds. And so it is quite natural to include these in the logical apparatus used to evaluate the dependence of a singleton on its member.

An alternative approach would be to take all of the impossible worlds, including those that are metaphysically and logically impossible, and any others there may be, and then reformulate the truth conditions for dependence in terms of a counterfactual conditional. Counterfactuals have traditionally been understood in terms of possible worlds. ${ }^{4}$ One looks to possible worlds that are relevantly similar to the actual world, but which make the antecedent true. If the consequent is also true at all of these worlds, then the whole counterfactual is true at the actual world. Adding impossible worlds to this framework, we could take the relevantly similar worlds to be those that are metaphysically impossible but not logically impossible. The truth conditions for dependence given in terms of counterfactuals would then be equivalent to those given here in terms of a strict conditional. For simplicity we restrict the impossible worlds under consideration and formulate the truth conditions in terms of a strict conditional.

Applying the revised truth conditions for dependence, we aim to show that, for any $x$ and $\{x\}$ that exist at the actual world, we have that at every world (either metaphysically possible or metaphysically impossible), if $x$ does not exist, then $\{x\}$ does not exist. We begin by giving a sketch of the

\footnotetext{
${ }^{4}$ See Lewis (1973) and Stalnaker (1968).
}

Australasian Journal of Logic (12:3) 2015, Article no. 2 
argument, filling in the technical details in the following section.

The argument proceeds by assuming that there is a world where $x$ does not exist, but $\{x\}$ does, and derives a contradiction. Supposing $x$ and $\{x\}$ exist at the actual world, we consider a world where $x$ does not exist but its singleton does. It is definitional of the singleton of $x$ that it is the set that contains $x$ and nothing else. That is, for every $y, y \in\{x\}$ iff $y=x$. As this is definitional of the singleton of $x$, it should hold at every world in this framework, including the actual world, and the world currently under consideration..$^{5}$ At this particular world, $x$ does not exist, and so nothing that exists at this world is identical to $x$. It follows that, at this world, the singleton of $x$ contains no members at all, i.e., it is identical to the empty set.

Next, we appeal to the necessity of identity, which says that if $x=y$, then at every world $x=y$. As a matter of logic, identity is generally taken to hold at every world. That is, the principle $x=y \rightarrow \square(x=y)$ is valid in all normal modal logics. Taking identity to be a logical notion, given that the worlds under consideration obey the laws of logic, this principle should still hold at those worlds that are metaphysically impossible. Even if one took identity to be more of a metaphysical notion than a logical one, for our purposes, there is no reason to question principles of identity at the impossible worlds we are interested in. We invoked impossible worlds to facilitate our desire to consider worlds that discriminate between objects that exist at all of the same possible worlds. Nothing about this project requires us to give up principles about the identity of those objects. Indeed, if we give up principles of identity, then it makes it difficult to ensure that we are talking about the same objects from one world to the next. If we want to say something substantive about $x$, we want to make sure that at each world under consideration, we are still talking about $x$.

If we do invoke the necessity of identity, it follows from the fact that $x=\varnothing$ at the world under consideration, that $x=\varnothing$ at every world, including the actual world. But at the actual world, or at any world, where $x$ exists, the singleton of $x$ is non-empty - it has $x$ as its single member. And thus we

\footnotetext{
${ }^{5}$ This stipulation is justified, even though we are considering metaphysically impossible worlds, because it ensures that we are talking about the singleton of $x$. If $\{x\}$ did not satisfy this condition, we would not be talking about the singleton of $x$; we would be talking about something else.
}

Australasian Journal of Logic (12:3) 2015, Article no. 2 
have a contradiction.

This argument makes several assumptions about the accessibility relation in the context of metaphysically possible and impossible worlds. Specifically, it requires that the accessibility relation of the modal logic under consideration is symmetric. Why should we think the accessibility relation symmetric? For one, metaphysical modality, where we consider only metaphysically possible worlds, has been taken by many to be correctly modelled by logics at least as strong as $55 .{ }^{6}$ Second, we want to consider what can happen at all worlds under consideration. This is properly done with a universal accessibility relation, according to which every world accesses every other world, and which is equivalent to S5. And the system S5 is characterised by having an accessibility relation that is not only symmetric, but also reflexive and transitive.

We have thus sketched an argument that, in every world under consideration, if $x$ does not exist, then $\{x\}$ does not exist, for any object $x$. On a natural reading, this tells us that you can't have $\{x\}$ without $x$. According to the revised truth conditions for dependence, we have that the singleton of an object depends on that object. But we need to do a bit more to show that this account is satisfactory. Because even on the standard modal account, which appeals only to possible worlds, we could show that $\{x\}$ depends on $x$. What we must also show is that this dependence is asymmetric, that $x$ does not also depend on $\{x\}$.

Using the same framework of worlds, which includes metaphysically possible and metaphysically impossible worlds, we want to show that there is some world where $x$ exists but $\{x\}$ does not. Recall that, in selecting the impossible worlds to include in our framework, a requirement that we enforced on these worlds was that they obey the laws of logic, including basic principles regarding identity. Given this selection of worlds, we have essentially shown that $\{x\}$ depends on $x$ in virtue of logical principles alone. But one cannot show through logic alone that in worlds where $\{x\}$ does not exist, $x$ must not exist either. And so one cannot show through logic alone that $x$ depends on $\{x\}$.

To make such a proof go through, one would need additional set-theoretic principles. For example, in the context of the iterative conception of set,

\footnotetext{
${ }^{6}$ See, for example, Lewis (1986) and Williamson (2013).
} 
and the Zermelo Fraenkel (ZF) axioms that formalise that conception, one must appeal to something like the pairing axiom, which says that for any two objects, $x$ and $y$, the pair set $\{x, y\}$ exists. Given the existence of some object, the pairing axiom implies that its singleton exists as well. ${ }^{7}$

If there are any (impossible) worlds where $x$ exists but $\{x\}$ does not, then pairing must fail at these worlds. Indeed, it must be that all of the relevant set-building principles fail. If these principles held at all of the worlds under consideration, then it would follow that each object depends on its singleton. And so the dependence between an object and its singleton would be symmetric, contrary to the view that dependence is an asymmetric relation. Consequently, such a result would be damaging to the extended truth conditions for dependence argued for here.

The question is then whether we can assume that these set-building principles hold at all of the relevant worlds. And we maintain that there doesn't seem to be any reason to assume that they do. These set-building principles are precisely the kind of principle that must fail in order to consider the relevant worlds where certain sets do not exist, i.e., the metaphysically impossible worlds. Given that we are trying to discover what happens when, e.g., the empty set or its singleton fail to exist, we cannot justify assuming principles that directly entail their existence.

In response, and recalling considerations from section 1 above, one might argue that the dependence of a set on its members only holds in the context of the iterative conception of set. And so we should only be looking at worlds where sets operate according to that conception. On that conception, these set-building principles hold. So we should only be considering worlds where these principles hold.

But by appealing to impossible worlds, where certain pure sets do not exist, one has already rejected the iterative conception of set. The iterative conception stipulates that certain sets exist, including the empty set and its singleton. By hypothesis, we are considering worlds where these sets fail to exist. And so we are considering worlds where sets do not operate according to the iterative conception. We should therefore be open to at least

\footnotetext{
${ }^{7}$ There are other ways besides pairing to construct the singleton of an object. This could be done, for example, with the power set axiom and an instance of the replacement axiom schema. For simplicity, we focus on pairing. But our remarks apply equally to these other set-generating axioms.
}

Australasian Journal of Logic (12:3) 2015, Article no. 2 
some worlds where certain set-building operations fail too. Rejecting the existence of certain sets is on a par with rejecting set-building principles.

Given the consideration of worlds where set-building principles fail, there is no reason to think that every world where $x$ exists must be a world where $\{x\}$ exists as well. And so we have an asymmetry. The existence of $x$ follows from the existence of $\{x\}$ though logic and definitions alone. All one needs is that, at the worlds under consideration, the laws of classical logic hold and we maintain the usual definitions or identity conditions for sets. The existence of $\{x\}$, however, does not follow from the existence of $x$ through logic alone - one needs extra set-theoretic principles to obtain at the worlds under consideration. Furthermore, it is precisely these kinds of principles which are in question, and so the burden is on our opponent to show why they must hold at every world.

The only set-theoretic axiom that seems plausible to maintain is the axiom of extensionality. Other axioms simply determine the existence of certain sets. Extensionality does not determine the existence of any sets at all; it determines the identity conditions for sets. It tells us that, at these worlds, we are really saying things about sets, and not some other kind of entity. In the details provided in the next section, we will appeal to the extensional nature of sets.

This assumption is not necessarily problematic. Extensionality describes one of the basic properties of the kind of objects set theory deals with. It tells us something fundamental about the nature of sets. Historically, the axiom of extensionality has been taken to be crucial to the concept of set, whereas other axioms can be called into question.

That the concepts of set and being a member of obey the axiom of extensionality is a far more central feature of our use of them than is the fact that they obey any other axiom. A theory that denied, or even failed to affirm, some of the other axioms of ZF might be called a set theory, albeit a deviant or fragmentary one. But a theory that did not affirm that the objects with which it dealt were identical if they had the same members would only by charity be called a theory of sets alone. (Boolos 1971, p. 28 , emphasis in original).

So one can deny the other ZF axioms and still claim to be talking about

Australasian Journal of Logic (12:3) 2015, Article no. 2 
sets. But to deny the extensionality of sets is to suggest that we are not really talking about sets at all.

\section{Filling in the Details}

In this section, we provide the details of the argument presented above that, in the framework that includes both metaphysically possible and metaphysically impossible worlds, there is no world where $\{x\}$ exists but $x$ does not, for any $x .{ }^{8}$ The language we use is the usual language of firstorder modal logic, including predicate symbols, variables, constants, connectives, quantifiers, identity, modal operators, and brackets. Terms and formulas are built up in the usual way.

The modal semantics we will deploy for this language is a standard constant domain semantics. An interpretation is a quadruple $\langle D, W, R, v\rangle$, where $D$ is the non-empty domain of quantification, $W$ is a non-empty set of worlds (that includes metaphysically possible and metaphysically impossible worlds), and $R$ is a binary accessibility relation on $W$. The function $v$ maps each constant, $c$, to a member, $v(c)$, of $D$. And to each pair consisting of a world, $w \in W$, and $n$-place predicate, $P$, the function $v$ assigns a subset of $D^{n}$ - the extension of $P$ at $w$, which we write as $v_{w}(P)$. We also reserve a one-place predicate symbol, $\mathcal{E}$, to serve as an existence predicate: if $\mathcal{E}(a)$ is true at $w$, then $a$ exists at $w$.

Truth conditions for the connectives, quantifiers, and modal operators are as usual. In particular, for atomic formulas we have:

$$
v_{w}\left(P a_{1} \ldots a_{n}\right)=\mathrm{T} \text { iff }\left\langle v\left(a_{1}\right), \ldots, v\left(a_{n}\right)\right\rangle \in v_{w}(P) \text { (otherwise it is } \mathrm{F} \text { ). }
$$

And for identity and existence we have the usual assignment:

$$
v_{w}(=) \text { is }\{\langle d, d\rangle: d \in D\} \quad v_{w}(\mathcal{E}) \subseteq D
$$

There is also one non-logical predicate, the two-place membership relation $\epsilon$. The only condition that this membership predicate must satisfy is the standard extensional identity condition:

$$
\forall x \forall y[\forall z(z \in x \leftrightarrow z \in y) \rightarrow x=y]
$$

\footnotetext{
${ }^{8}$ Here we follow the notation of Priest (2008).
}

Australasian Journal of Logic (12:3) 2015, Article no. 2 
Given this framework, we aim to show that, for any object, if both that object and its singleton exist, then, in every world, if that object does not exist, then its singleton does not exist. Choose an arbitrary object and assign it the constant symbol $a$. We have at our base world, $w$, that $a$ exists, and that it's singleton, which we label $s$, exists too.

(1) $w \Vdash \mathcal{E}(a)$

(2) $w \Vdash \mathcal{E}(s)$

The singleton of $a$ is the set of all things at $w$ that are identical to $a$. So we have:

(3) $w \Vdash \forall x[x \in s \leftrightarrow(\mathcal{E}(x) \wedge x=a)]$

And so we have that $a \in s$. Given the extensional nature of sets, we also have that anything at $w$ that is the singleton of $a$ is identical to $s$.

(4) $w \Vdash \forall y[\forall x[x \in y \leftrightarrow(\mathcal{E}(x) \wedge x=a)] \rightarrow y=s]$

In this sense, we take the condition $x \in y \leftrightarrow(\mathcal{E}(x) \wedge x=a)$ to be definitional of the singleton of $a$. Any $y$, in any world, that satisfies this condition is the singleton of $a$.

We want to show, at $w$, that at every world, if $a$ does not exist, then it's singleton does not exist. We assume the opposite and derive a contradiction.

(5) $w \Vdash \diamond \neg[\neg \mathcal{E}(a) \rightarrow \neg \exists y \forall x[x \in y \leftrightarrow(\mathcal{E}(x) \wedge x=a)]]$

So there is a world, $w^{\prime} \in W$, such that $w R w^{\prime}$. From (5) it follows that:

(6) $w^{\prime} \Vdash \neg \mathcal{E}(a)$

(7) $w^{\prime} \Vdash \exists y \forall x[x \in y \leftrightarrow(\mathcal{E}(x) \wedge x=a)]$

Line (7) says there exists something at $w^{\prime}$ that has $x$ as a member iff $x$ exists at $w^{\prime}$ and $x=a$. Take this to be a set, and call it $s^{\prime}$. It isn't hard to see that, at $w^{\prime}, s^{\prime}$ is the empty set. For suppose that there exists an $x$ such that $x \in s^{\prime}$. It follows that $x=a$ and that $\mathcal{E}(x)$. By substitution we would have that $\mathcal{E}(a)$, but by (6) we have $\neg \mathcal{E}(a)$. So $s^{\prime}$ is empty.

(8) $w^{\prime} \Vdash \neg \exists x\left(x \in s^{\prime}\right)$

It is at this point that we invoke the necessity of identity, whereby if $x=y$, then $\square(x=y)$. As $s^{\prime}=\varnothing$ at $v$, we have that $\square\left(s^{\prime}=\varnothing\right)$ at $v$. One can 
justify this inference by observing that both $s$ and $\varnothing$ are names. And so we have reason to believe they refer rigidly, to the same object in every world. ${ }^{9}$ As long as the accessibility relation is symmetric, from $w R w^{\prime}$, we have $w^{\prime} R w$, and so $s^{\prime}=\varnothing$ at $w$. We gave reason in section 3 to think that the accessibility relation corresponds to that of the modal logic S5, which makes it an equivalence relation, and thus symmetric. It follows that:

(9) $w \Vdash \neg \exists x\left(x \in s^{\prime}\right)$

But recall that $s^{\prime}$ is defined as the set that has as its members all and only those objects that are identical to $a$. As this is definitional of $s^{\prime}$, it should hold in every world, including $w$.

(10) $w \Vdash \forall x\left[x \in s^{\prime} \leftrightarrow(\mathcal{E}(x) \wedge x=a)\right]$

We have from (4) and (10) that $s^{\prime}=s$. By extensionality, it follows that $a \in s^{\prime}$, but by (9) it follows that $a \notin s^{\prime}$. And so we have our contradiction. It follows on this framework that, for any $x$, if $x$ and its singleton exist at a world $w$, then, in every world $w^{\prime}$, if $x$ doesn't exist at $w^{\prime}$, then it's singleton doesn't exist at $w^{\prime}$ either. According to the revised modal truth conditions, that allow consideration of both metaphysically possible and metaphysically impossible worlds, it follows that for any object $x$, the singleton of $x$ depends on $x$.

It isn't too hard to show that the converse does not hold, and so dependence on this framework is asymmetric. Consider the interpretation where $W$ contains two worlds, $w$ and $w^{\prime}$, the domain of $w$ has both $a$ and its singleton, and the domain of $w^{\prime}$ has just $a$. In this interpretation, we have:

(11) $w \Vdash \diamond[\mathcal{E}(a) \wedge \neg \exists y \forall x[x \in y \leftrightarrow(\mathcal{E}(x) \wedge x=a)]]$

That is, there is a world such that $a$ exists while its singleton does not. The only way to falsify this statement would be to invoke certain set-building principles, such as those captured by the pairing axiom. But as discussed in section 3, given the kind of worlds under consideration, we cannot assume that these principles hold at all worlds. And so they may fail at some particular world, thus making (11) true.

\footnotetext{
${ }^{9}$ See Kripke (1972). In fact, the rigidity of the names is equivalent to validity of the inference. See Fitting \& Mendelsohn (1998).
}

Australasian Journal of Logic (12:3) 2015, Article no. 2 


\section{Conclusion}

We have argued in favour of a framework that can be used to understand and evaluate statements of dependence between sets and their members. We have shown that, within this framework, a singleton depends on the single object that is its member, and that this dependence is asymmetric - the single member does not depend on its singleton. There is reason to think that this framework can be used to evaluate more general claims that sets depend on their members. There is also reason to think that the argument presented here can be generalised to show that, beyond the case of singletons, all sets depend on their members, and that this dependence is asymmetric.

This framework has been presented in the form of a normal first-order modal logic. In the technical details, very little is required of the worlds that provide the semantics for the modal language that is used. They obey the laws of classical logic, they satisfy the extensional identity conditions that are standard for sets, and they maintain the standard definition for singletons. As we are interested in the dependence that holds between sets and their members, these requirements are appropriate. They ensure that, when we claim to be talking about sets in this framework, we actually are talking about sets.

Though very few assumptions are made about these worlds from a formal perspective, one could ask what these worlds represent. A philosophical treatment of these worlds would take them to include both metaphysically possible and metaphysically impossible worlds, as some worlds under consideration must make it that certain necessarily existing objects, like pure mathematical objects, fail to exist. But these worlds will not be logically impossible, as they will continue to obey the laws of classical logic.

Given that the formal details require very little of the worlds under consideration, one could argue that what we have shown is that the dependence of a singleton on its members follows from logical principles alone, once we have the appropriate logical framework in place to understand this kind of dependence claim. As long as we hold fixed certain definitions, such as the definition of singleton, and what it means for two sets to be identical, logic determines that, in any world, $w$, if an object does not exist

Australasian Journal of Logic (12:3) 2015, Article no. 2 
at $w$, then its singleton doesn't exist at $w$ either.

However, logic alone is not enough to establish the dependence of an object on its singleton. One must invoke further set-theoretic principles in order to establish this dependence. But these principles go beyond standard principles of logic. And so, as desired, we have an asymmetric dependence relation between singletons and their members: singletons depend on their (single) members, while those members do not depend on their singletons.

\section{References}

Aczel, P. (1988). Non-Well-Founded Sets. Stanford: Center for the Study of Language and Information.

Barwise, J., \& Moss, L. (1996). Vicious Circles: On the Mathematics of NonWellfounded Phenomena. Stanford: Center for the Study of Language and Information.

Boolos, G. (1971). The iterative conception of set. The Journal of Philosophy 68, 215 - 232. Reprinted in Boolos (1998), pages 13 - 29.

Boolos, G. (1998). Logic, Logic, and Logic. Cambridge, MA: Harvard University Press.

Cameron, R. (2008). Turtles all the way down: Regress, priority and fundamentality. The Philosophical Quarterly 58, 1-14.

Correia, F. (2005). Existential Dependence and Cognate Notions. Munich: Philosophia Verlag.

Fine, K. (1995). Ontological dependence. Proceedings of the Aristotelian Society 95, $269-290$.

Fitting, M., \& Mendelsohn, R. L. (1998). First-Order Modal Logic. Dordrecht: Kluwer Academic Publishers.

Hoelte, M., Schnieder, B., \& Steinberg, A. (2013). Varieties of Dependence. Munich: Philosophia Verlag.

Australasian Journal of Logic (12:3) 2015, Article no. 2 
Incurvati, L. (2012). How to be a minimalist about sets. Philosophical Studies 159, $69-87$.

Kripke, S. (1972). Naming and Necessity. Cambridge, MA: Harvard University Press.

Lewis, D. (1973). Counterfactuals. Cambridge, MA: Harvard University Press.

Lewis, D. (1986). On the Plurality of Worlds. Oxford: Basil Blackwell.

Lowe, E. J. (2005). Ontological dependence. Stanford Encyclopedia of Philosophy, http:/ / plato.stanford.edu/entries/dependence-ontological/.

Paseau, A. (2010). Defining ultimate ontological basis and the fundamental layer. Philosophical Quarterly 60, 169 - 175.

Potter, M. (2004). Set Theory and its Philosophy: A Critical Introduction. Oxford: Oxford University Press.

Priest, G. (2008). An Introduction to Non-Classical Logic: From If to Is, second edition. Cambridge: Cambridge University Press.

Rescher, N., editor (1968). Studies in Logical Theory, American Philosophical Monograph Series, no 2. Oxford: Basil Blackwell.

Schaffer, J. (2010). Monism: The priority of the whole. Philosophical Review 119, $31-76$.

Schnieder, B. (2007). Review of Correia Existential Dependence and Cognate Notions. Dialectica 61, 589 - 594.

Stalnaker, R. (1968). A theory of conditionals. In Rescher (1968), pp. 98 112.

Trogdon, K. (2013). An introduction to grounding. In Hoelte et al. (2013), pp. $97-122$.

Williamson, T. (2013). Modal Logic as Metaphysics. Oxford University Press.

Australasian Journal of Logic (12:3) 2015, Article no. 2 